\title{
Evolution of sinonasal radiological findings in a case of granulomatosis with polyangiitis
}

\author{
Aru Chhabra Handa ${ }^{1}$, Anup Singh ${ }^{1}$, Jai Prakash Sharma² \\ ${ }^{1}$ Department of Otolaryngology and Head and Neck Surgery, Medanta - The Medicity, Gurugram, India \\ ${ }^{2}$ Division of Radiology and Nuclear Medicine, Medanta - The Medicity, Gurugram, India
}

\begin{abstract}
Wegener's granulomatosis (Granulomatosis with Polyangiitis) is an autoimmune granulomatous disorder of unknown etiology. The basic pathology is necrotizing granulomatous inflammation with necrotizing vasculitis involving medium-sized blood vessels. The typical triad of involvement of the upper and lower respiratory tracts and the kidneys is observed in varying proportions in individual patients. The sinonasal involvement is observed in the majority of patients and it can be involved in isolation in up to a quarter of affected individuals. The sinonasal changes involve both the soft tissue and bony architecture, with refractory chronic sinusitis associated with erosion of the cartilaginous and bony skeleton and may lead to saddle nose and other facial deformities in advanced cases. The radiology has a contributory role as diagnostic and diseases monitoring aid.

The various radiological findings associated with the disease (bone erosion with osteoneogenesis) have been mentioned in the literature. We present the progression of a radiological abnormality in a patient with a localized form of Wegener's Granulomatosis and highlight some peculiar appearances observed on radiology in the patient.
\end{abstract}

KEYWORDS: Wegener's Granulomatosis, chronic rhinosinusitis, radiology, osteoneogenesis.

\section{INTRODUCTION}

Wegener's granulomatosis (Granulomatosis with polyangiitis $\{\mathrm{GPA}\})$ is a rare autoimmune disease characterized by necrotizing vasculitis of small and medium-sized vessels, manifesting as upper and lower airway symptoms and renal dysfunction, associated with c-ANCA (cytoplasmic anti-neutrophil cytoplasmic antibody) positivity.

Three types of GPA are recognized based on the pattern of organ involvement: type 1 - the classic generalized form with the systemic involvement of the lungs and kidneys; type 2 - the localized form without renal involvement and type 3 - the purely granulomatous (without evidence of vasculitis), localized Wegener's granulomatosis (head and neck involvement $)^{1}$. The various head and neck subsites, in particular the sinonasal areas, are the most com- mon sites of involvement by GPA. The diagnosis of GPA relies on characteristic histopathological findings and it is supported by serological findings. Radiology has an important role in defining the extent of involvement by the disease and in disease monitoring during the follow-up. Certain radiological findings may point to the underlying granulomatous pathology and help in narrowing down the list of differential diagnoses.

The cross-sectional radiological appearance of sinonasal involvement with GPA has been previously described in the literature. We discuss the radiological progression of the sinonasal disease in a case of type 2 GPA (the limited form) with the involvement of the lungs and sinonasal region, with the emphasis on bony and soft tissue changes overtime. A peculiar associated finding of 'Hyperostosis Frontalis Interna' (HFI) is also described. 


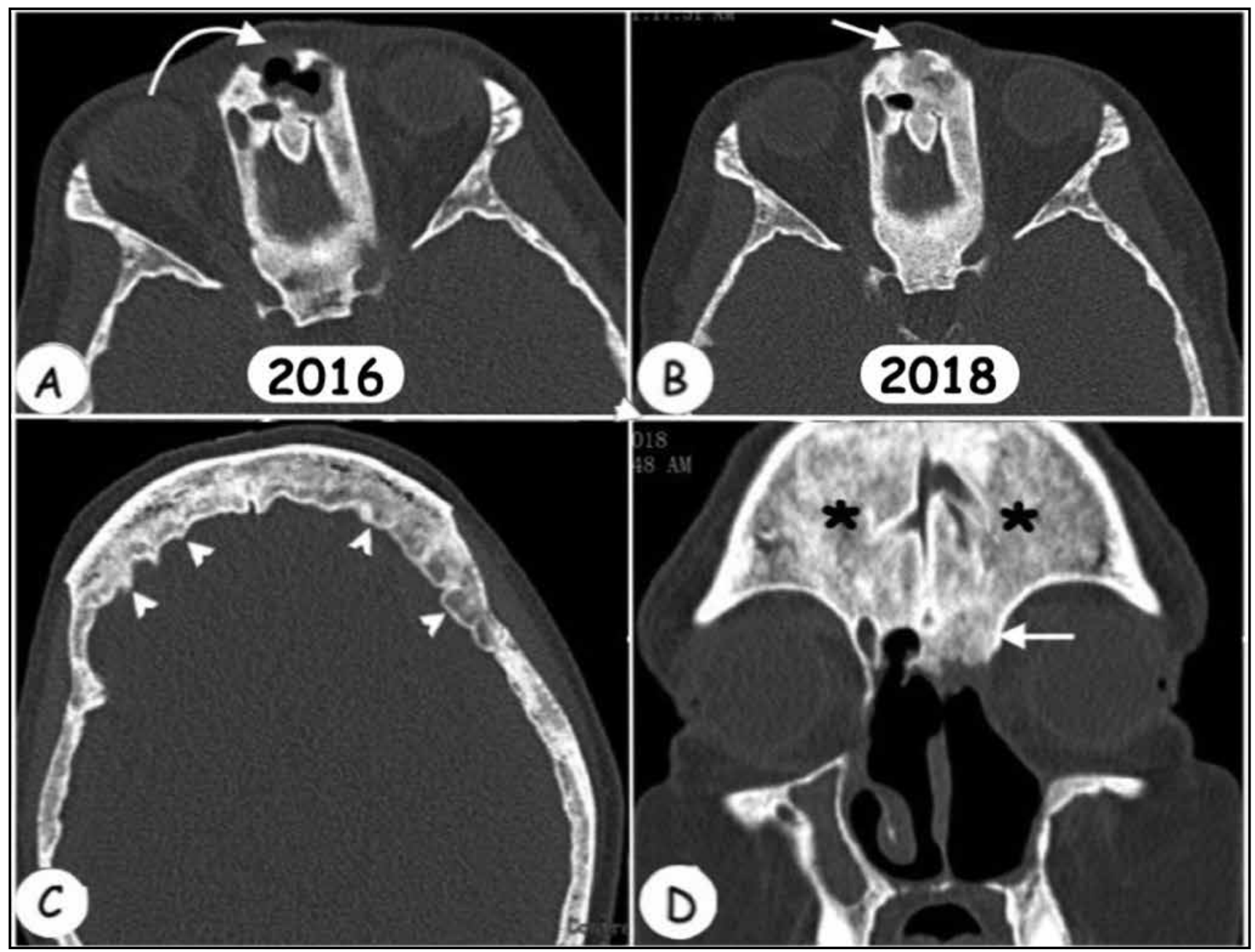

Figure 1 Axial (A, B and C) and coronal (D) views at the level of the frontal sinus showing diffuse sclerosis of the frontal sinus $\left({ }^{*}\right.$ in $\left.D\right)$ along with erosion of the anterior wall of the infundibulum of the frontal sinus (curved arrow in A). The encroachment of the sclerotic changes in the region of the frontal infundibulum and frontal recess (straight arrows) is depicted in Figures B and D. The characteristic nodular bony hypertrophy (arrowheads) of the hyperostosis frontalis interna (HFI) can be seen in Figure C.

\section{CASE REPORT}

A 71-year-old female presented to us with a history of recurrent upper respiratory tract infections since childhood. She had had antral punctures twice in childhood. In 2002, she underwent resection of an anterior mediastinal mass lesion under general anaesthesia at another center. Post-operative histopathology revealed a yellowish lung nodule with necrotizing granulomata, suggestive for Wegener's granulomatosis. The stains for the Acid Fast Bacilli (AFB) and Fungus were negative. After surgery, her Erythrocyte Sedimentation Rate (ESR) was raised $(62 \mathrm{~mm})$. There were no other systemic features and the antinuclear antibody (ANA), the cytoplasmic anti-neutrophil cytoplasmic antibody (c-ANCA), the perinuclear anti-neutrophil cytoplasmic antibody (p-ANCA), the rheumatoid factor $(\mathrm{RF})$, urinalysis and the urine microscopy were within normal limits. No medical management with steroids or cyclophosphamide was given.

The patient remained in follow-up without any further symptoms (except an episode of acute rhinosinusitis in 2010, which subsided with conservative management) for the next 14 years, when in 2016, she presented to us with nasal stuffiness and occasional mild spontaneous nasal bleed. She gave a positive history of hyposmia. There was no history of snoring / facial pain / toothache or headaches. There were no ear / throat or ocular symptoms. The nasal examination showed a roomy nasal cavity with extensive crusting and focal areas of congested and ulcerated mucosa. Crusts were removed and nasal endoscopic examination showed two perforations involving the bony septum with significantly atrophied turbinates. The examination of the ear / oral cavity / throat / larynx / neck and eyes was 


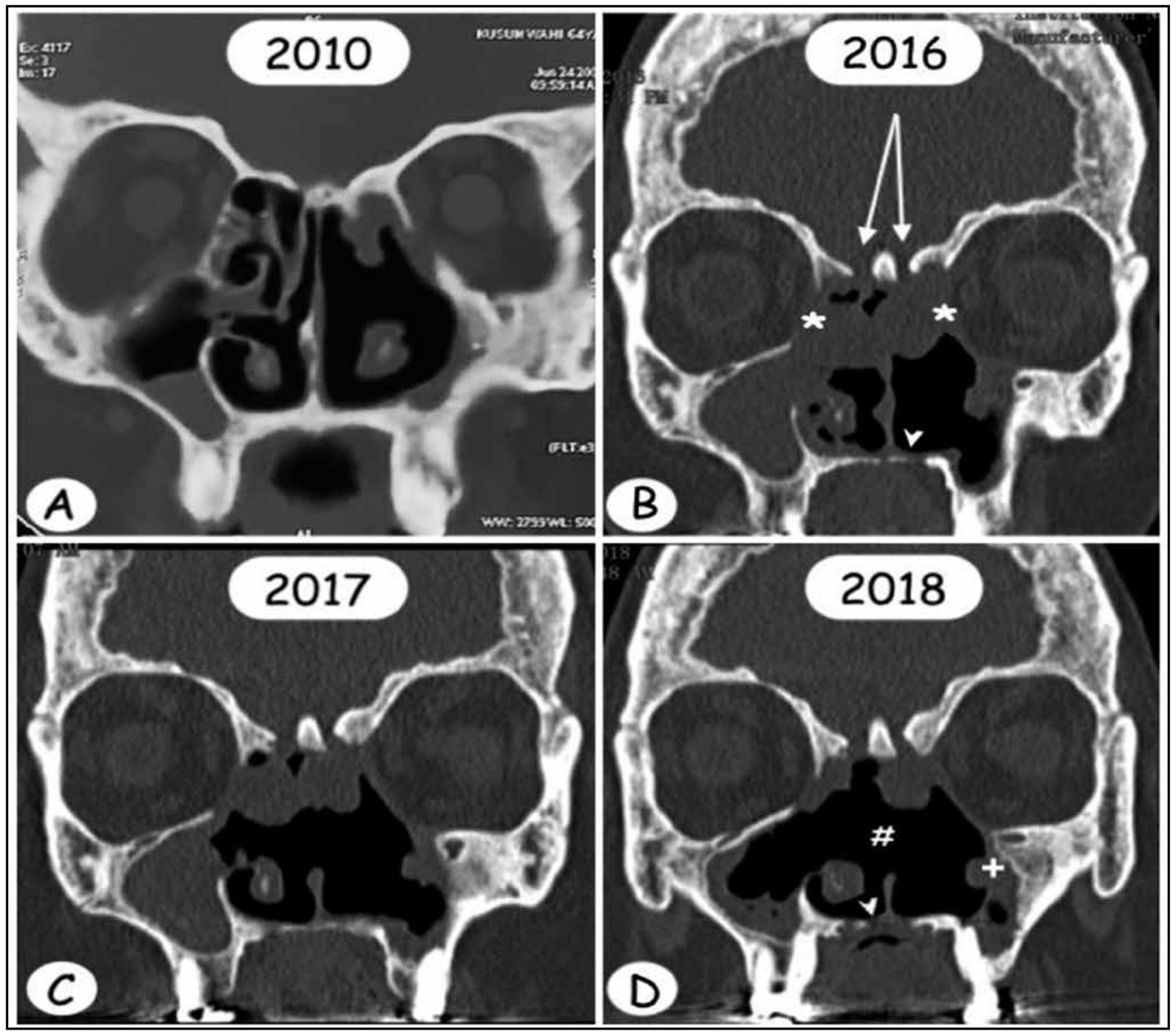

Figure 2 Non-contrast computed tomography of the paranasal sinuses at midnasal area showing the destruction of the lateral nasal wall bilaterally (Lt $>\mathrm{Rt}$ ). Destruction of the bilateral lamina papyracea ${ }^{*}$ ) and defect in the horizontal lamella of the cribriform plate (arrows) on both the sides is also seen (B-D). Complete loss of the ethmoid trabeculae, progressive destruction of the nasal septum (\#) and defects in the hard palate (arrowheads) can be seen in serial scans (B-D). Interval increase in the thickening and sclerosis of the posterolateral wall of the left maxillary sinus can be seen (B-D). Neocortical bone deposition with underlying rarefied bone deposition can be seen in Fig D ('+').

within normal limits. A Non-Contrast Computed Tomography of paranasal sinuses (NCCT PNS) was obtained and it showed diffuse soft tissue density with extensive bony destruction involving the bony septum, the turbinates and lamina papyracea (Figures 2B and 3B). High-Resolution Computed Tomography (HRCT) of the chest showed no evidence of active disease focus. Urinalysis and urine microscopy results were normal. Repeated c-ANCA levels showed weak positivity on one occasion; however, the same could not be reproduced with repeat testing and during follow-up.

She was started on alkaline nasal douching and glucose / glycerine solution nasal drops. Oral Prednisolone was started in a dose of $60 \mathrm{mg} /$ day for one month and then tapered over two months to $2.5 \mathrm{mg} /$ day. Oral methotrexate (MTX) was started in a dose of $20 \mathrm{mg} /$ week. C-reactive protein was found to be raised on one occasion $(18 \mathrm{mg} / \mathrm{L}$; normal $<10 \mathrm{mg} / \mathrm{L})$ and came out to be normal at the rest of the follow-up visits. ESR was mildly raised in the range of $30-40 \mathrm{~mm}$ during the follow-up visits. Nasal endoscopic cleaning was continued every few months in the outpatient department. An NCCT PNS done in 2018 showed the persisting bone remodelling changes, but the sinonasal soft tissue 


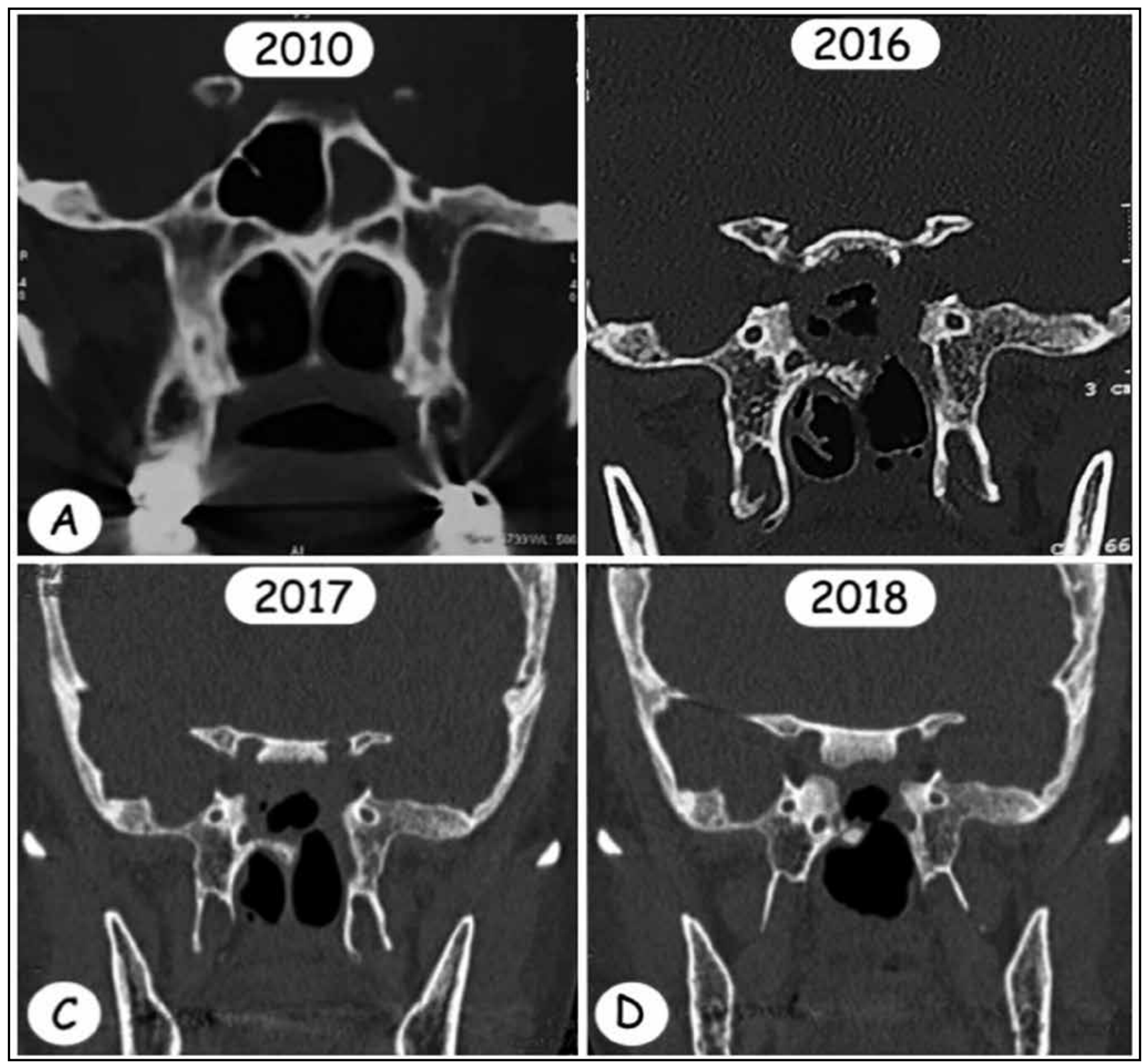

Figure 3 Non-contrast computed tomography of the paranasal sinus at the level of the sphenoid sinus showing the destruction of the lateral walls and the left side of the floor of the sphenoid sinus, with increasing sclerosis of the residual walls of the sinus on serial scans (A-D).

density had significantly regressed (Figures 2D and 3D). The serial bony and soft tissue changes are depicted in Figures 1, 2 and 3. Significant bone erosion co-existent with sclerosis and osteoneogenesis can be appreciated. Figure 1C shows nearly complete sclerosis of the frontal sinus with nodular thickening of the endocranial aspect of the frontal bone. There is only mild crusting and the mucosa has reverted to a normal appearance on examination (Figure 4). In view of a well-controlled disease, she is being contemplated to stop the oral steroids / MTX and continue alkaline nasal douching to prevent crusting associated with atrophic rhinitis and septal perforation.

\section{DISCUSSIONS}

Wegener's granulomatosis, now called granulomatosis with polyangiitis (GPA), was first described in the 1930s by Klinger and later proposed and elaborated by Wegener. In the initial days of description, the mortality from the disease reached as high as $82 \%$ by one year and more than $90 \%$ by 2 years. The mean survival rate was only $\sim 5$ months. In 1954, the addition of Cyclophosphamide was suggested and today, with the combination of steroid with Cyclophosphamide or Rituximab, mortality has been brought down substantially, with a post-diagnosis median survival time of 21.7 years $^{2,3}$. 


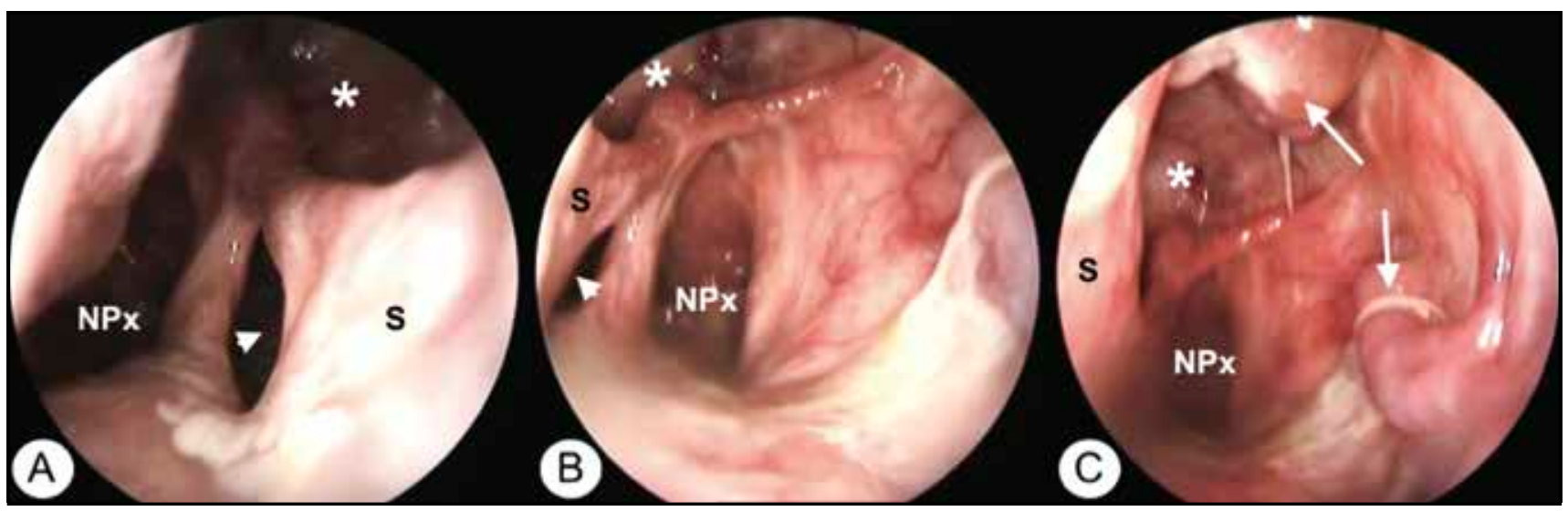

Figure 4 Endoscopic view of the nasal cavity showing: (A) the right nasal cavity, (B) the left nasal cavity and (C) the left lateral nasal wall. (Asterisk - septal perforation involving the perpendicular plate of the ethmoid; arrow heads - septal perforation involving the vomer bone; white arrows atrophic inferior and middle turbinates; S - nasal septum; NPx - nasopharynx).

Methotrexate, instead of cyclophosphamide, is also used in localized disease without systemic involvement ${ }^{3}$. Our patient, who had localized nasal and paranasal involvement with the disease, was also managed with a combined therapy of steroid and methotrexate. Head and neck involvement is usually associated with a good prognosis in terms of survival, but the relapse rate is higher compared to the systemic variant ${ }^{4}$. The relapse rate can be reduced by early immunosuppression.

Head and neck region involvement in GPA is seen in up to $85 \%$ of cases $^{5}$. The most common site of involvement in the head and neck region is the sinonasal area and isolated sinonasal involvement can be seen in up to $25 \%$ of cases. The diagnosis is based on clinical, histopathological and laboratory findings. However, the diagnostic possibility is usually overlooked clinically, there being no pathognomonic signs in the early stage. Histopathology from the nose biopsy showing characteristic granulomatous inflammation and necrotising vasculitis is diagnostic and it is seen in up to $53 \%$ of cases ${ }^{6}$. cANCA are the antibodies against the neutrophil / monocyte cytoplasmic protein, Proteinase-3 (PR3 ), described first by Van Der Woude in $1985^{7}$. The specificity of c-ANCA as a diagnostic marker is $98 \%$, but the sensitivity varies as per the stage of the disease. Serology can also serve to titrate the response to therapy and as an indicator of disease relapse; however, the gold standard for diagnosis remains the biopsy ${ }^{8}$.

Radiology can help in providing additional information regarding the extent of the disease, can aid in the diagnosis especially in ANCA negative cases and guide in preoperative planning, as well as follow-up of disease stage / activity. Only one in ten patients with GPA has been reported to have normal imaging findings. The spectrum of typical sinonasal findings in GPA includes mucosal thickening ( $88 \%$ of cases), most commonly involving the maxillary sinuses, to osseous erosion $(\sim 60 \%$ of cases) and osteoneogenesis $(\sim 56 \% \text { of cases })^{9}$. $\mathrm{Al}$ though these changes can occur in garden-variety chronic rhinosinusitis also, there are certain subtle indicators which indicate a possibility of underlying GPA. The mucosal thickening in GPA tends to be 'nodular' in appearance.

The bone erosions, due to avascular necrosis, seen in and around the nose in GPA, tend to be more extensive and deeper, leading to septal perforations and resorption of the lateral nasal wall in severe cases. Large septal perforations also tend to cause saddle deformity of the nose. In our patient, we saw progressive osseous destruction over the years, involving the nasal septum, turbinates, lamina papyracea, lateral wall of the nose, frontal bone, ethmoid, cribriform plate, sphenoid and palate (Figures 1-3). Lloyd et al. ${ }^{10}$, in their retrospective review of 28 patients, did not find palatal erosion in any of the scans; however, it was demonstrated in our present case. This case had extensive erosion of the lateral wall of the sphenoid sinus and the serial coronal CT scans showed decrease in the height of the sinus because of the erosion and osteoneogenesis.

Bone sclerosis is another common finding and it is probably due to periosteal inflammation and thickening leading to new bone formation, due to vasculitis or secondary to chronic sinus infection. A combination of osseous erosion with osteoneogenesis without previous surgery points towards involvement with a granulomatous pathology. Another characteristic finding that points towards involvement with GPA is the neo-osteogenesis seen as a line of new cortical bone formation along the sinus wall and an area of rarefaction representing 
bone marrow deposition between this new cortical bone and the parallel original sinus cortical bone $\mathrm{e}^{10,11}$. In our patient this finding was absent in the first CT scan done in 2010 but subsequent CT scans done after 2016 showed this changes in the left antral wall (Figure 2A-D). Our patient incidentally also showed the presence of asymptomatic hyperostosis frontalis interna (HFI), with nodular thickening of the endocranial aspect of the frontal bone. To the best of our knowledge, the association of this peculiar feature with GPA has not been described in the literature hitherto. HFI is more commonly seen in menopausal women above 50 and is possibly due to hormonal influence on skull bone growth ${ }^{12}$.

\section{CONCLUSIONS}

This case report highlights the dynamic serial radiological changes in the sinonasal compartment in a patient with a protracted course of type 2 (limited) Wegener's granulomatosis. The overall look on the CT scan gives the impression of postoperatory changes in a chronic sinusitis case. However, absence of history of surgery with such changes should raise the suspicion of GPA and warrant further investigation. Furthermore, in this case, an association between HFI and GPA has been reported for the first time in the literature.

Informed/ Written consent: It was obtained from the individuals participating in the study.

Conflict of interests: The author reports no conflicts of interest.

Funding: This research did not receive any specific grant from funding agencies in the public, commercial, or not-for-profit sectors.

Acknowledgements: none declared.

\section{REFERENCES}

1. Boudes P. Purely granulomatous Wegener's granulomatosis: a new concept for an old disease. Semin Arthritis Rheum. 1990;19(6):365-70.

2. Reinhold-Keller E, Beuge N, Latza U, de Groot K, Rudert H, Nölle B, et al. An interdisciplinary approach to the care of patients with Wegener's granulomatosis: long-term outcome in 155 patients. Arthritis Rheum. 2000;43(5):1021-32.

3. De Groot K, Rasmussen N, Bacon PA, Tervaert JW, Feighery C, Gregorini G, et al. Randomized trial of cyclophosphamide versus methotrexate for induction of remission in early systemic antineutrophil cytoplasmic antibody-associated vasculitis. Arthritis Rheum. 2005;52(8):2461-9.

4. Bligny D, Mahr A, Toumelin PL, Mouthon L, Guillevin L. Predicting mortality in systemic Wegener's granulomatosis: a survival analysis based on 93 patients. Arthritis Rheum. 2004;51(1):83-91.

5. McDonald TJ, DeRemee RA. Wegener's granulomatosis. Laryngoscope. 1983;93(2):220-31.

6. Del Buono EA, Flint A. Diagnostic usefulness of nasal biopsy in Wegener's granulomatosis. Hum Pathol. 1991;22(2):107-10.

7. Van der Woude FJ, Rasmussen N, Lobatto S, Wiik A, Permin H, van Es LA, et al. Autoantibodies against neutrophils and monocytes: tool for diagnosis and marker of disease activity in Wegener's granulomatosis. Lancet. 1985;1(8426):425-9.

8. Helmberger RC, Mancuso AA. Wegener granulomatosis of the eustachian tube and skull base mimicking a malignant tumor. AJNR Am J Neuroradiol. 1996;17(9):1785-90.

9. D'Anza B, Langford CA, Sindwani R. Sinonasal imaging findings in granulomatosis with polyangiitis (Wegener granulomatosis): A systematic review. Am J Rhinol Allergy. 2017;31(1):16-21. DOI: 10.2500/ ajra.2017.31.4408.

10. Lloyd G, Lund VJ, Beale T, Howard D. Rhinologic changes in Wegener's granulomatosis. J Laryngol Otol. 2002;116(7):565-9.

11. Pakalniskis MG, Berg AD, Policeni BA, Gentry LR, Sato Y, Moritani T, et al. The many faces of granulomatosis with polyangiitis: a review of the $\underline{\text { head }}$ and neck imaging manifestations. AJR Am J Roentgenol. 2015;205(6):W619-29. DOI: 10.2214/AJR.14.13864.

12. Hershkovitz I, Greenwald C, Rothschild BM, Latimer B, Dutour O, Jellema LM, et al. Hyperostosis frontalis interna: an anthropological perspective. Am J Phys Anthropol. 1999;109(3):303-25. 